OPEN ACCESS

Edited by:

Carmen Rondon,

Regional University Hospital of

Malaga, Spain

Reviewed by:

Cristobal Langdon Montero, Hospital Clínic de Barcelona, Spain

Yuji Nakamaru,

Hokkaido University, Japan

${ }^{*}$ Correspondence:

Jonathan A. Bernstein

jonathan.bernstein@uc.edu

Specialty section:

This article was submitted to

Rhinology,

a section of the journal

Frontiers in Allergy

Received: 06 July 2021

Accepted: 24 August 2021

Published: 01 October 2021

Citation:

Huntley KS, Raber J, Fine $L$ and Bernstein JA (2021) Influence of the Microbiome on Chronic Rhinosinusitis With and Without Polyps: An Evolving

Discussion. Front. Allergy 2:737086.

doi: 10.3389/falgy.2021.737086

\section{Influence of the Microbiome on Chronic Rhinosinusitis With and Without Polyps: An Evolving Discussion}

\author{
Kyle S. Huntley ${ }^{1}$, Joshua Raber ${ }^{1}$, Lauren Fine ${ }^{1}$ and Jonathan A. Bernstein ${ }^{2 *}$ \\ ${ }^{1}$ Dr. Kiran C. Patel College of Allopathic Medicine, Nova Southeastern University, Fort Lauderdale, FL, United States, \\ ${ }^{2}$ Department of Internal Medicine, Division of Immunology/Allergy Section, University of Cincinnati College of Medicine, \\ Cincinnati, $\mathrm{OH}$, United States
}

Chronic rhinosinusitis (CRS) is widely prevalent within the population and often leads to decreased quality of life, among other related health complications. CRS has classically been stratified by the presence of nasal polyps (CRSwNP) or the absence nasal polyps (CRSsNP). Management of these conditions remains a challenge as investigators continue to uncover potential etiologies and therapeutic targets. Recently, attention has been given to the sinunasal microbiota as both an inciting and protective influence of CRS development. The healthy sinunasal microbiologic environment is largely composed of bacteria, with the most frequent strains including Staphylococcus aureus, Streptococcus epidermidis, and Corynebacterium genera. Disruptions in this milieu, particularly increases in S. aureus concentration, have been hypothesized to perpetuate both Th1 and Th2 inflammatory changes within the nasal mucosa, leading to CRS exacerbation and potential polyp formation. Other contributors to the sinunasal microbiota include fungi, viruses, and bacteriophages which may directly contribute to underlying inflammation or impact bacterial prevalence. Modifiable risk factors, such as smoking, have also been linked to microbiota alterations. Research interest in CRS continues to expand, and thus the goal of this review is to provide clinicians and investigators alike with a current discussion on the microbiologic influence on CRS development, particularly with respect to the expression of various phenotypes. Although this subject is rapidly evolving, a greater understanding of these potential factors may lead to novel research and targeted therapies for this often difficult to treat condition.

Keywords: chronic rhinosinusitis, nasal polyp, allergic rhinitis, non-allergic rhinitis, microbiota

\section{INTRODUCTION}

The human microbiota plays a crucial role in the preservation of human health, and investigations into this complex ecosystem have become a key focus in medical research (1). Defined as a community of microorganisms within a specific environment, the microbiota generally serves a mutualistic purpose in the human body; organisms gain a nourishing milieu while the human host receives benefits such as improved immunologic development and metabolic function (2-4). However, when disturbed, this previously mutualistic symbiosis can transform into a 
destructive process, with consequences affecting multiple organ systems. A prominent example of this phenomenon is the gutlung axis, whereby disturbances of the intestinal microbiota are implicated in pathologic changes of the respiratory system $(5,6)$. Specifically, decreases in gut bacterial diversity have been hypothesized to shift innate immunologic responses from a Th1 to a Th2 predominance, leading to atopic disease potentiation, including asthma and allergic rhinitis $(7,8)$. Discovery of these microbiologic connections have spurred investigations into previously unexplored microbiologic milieu, including the upper airway, and their contributions toward disease (5).

Upper airway diseases that are now hypothesized to be linked to microbiologic processes include rhinitis and chronic rhinosinusitis (CRS) (9). Rhinitis represents a heterogenous syndrome which may be acute or chronic, and is characterized by at least one of the following symptoms: nasal congestion, rhinorrhea (anterior and posterior), sneezing, and nasal itching (10). Rhinitis can additionally be classified into allergic (AR) or non-allergic (NAR) subtypes which are differentiated by clinical history and the presence of an IgE-mediated inflammatory reaction in response to allergen exposure. In contrast, CRS is defined as a clinical syndrome classified by at least 12 weeks of inflammation of the sinunasal mucosa which results in facial pain and/or pressure, hyposmia/anosmia, nasal drainage, and nasal obstruction $(11,12)$. This latter syndrome is classified into two subtypes, CRS with nasal polyps (CRSwNP) and CRS without nasal polyps (CRSsNP), each with diverse pathologic mechanisms (13). CRS is widely prevalent within the US and Europe and thus has a significant socioeconomic and quality of life impact (14). Although there is some overlap between the clinical manifestations of pure rhinitis (particularly AR) and CRS, each are driven by differing pathologic mechanisms and potential influences of the local microbiota. Definitive treatment and understanding of CRS remain elusive, but recent technological advances in microbiologic characterization have highlighted both the protective and pathogenetic roles of the nasopharyngeal and sinus microbiotas. A greater microbiologic understanding of CRS can guide clinicians toward more focused treatment and encourage future therapeutic research efforts.

Here, we provide a broad overview of the current pathophysiologic understanding of both CRSwNP and CRSsNP and how microbiologic influences in resident bacteria, fungi, and viruses, may contribute to disease formation. Because this subject matter is rapidly evolving, the purpose of this review is to provide a thorough discussion on the nasopharyngeal and sinus microbiotas regarding risk factors, current treatments, and future therapeutic directions.

\section{Pathogenesis of CRS With and Without Nasal Polyps}

Various theories regarding the underlying pathogenesis of CRS exist, including underlying host responses to airborne fungal elements, superantigenic exotoxins produced by Staphylococcus, pro-inflammatory biofilms, interruption of the sinunasal microbiome, and defects in the host barrier with inappropriate immune responses (Figure 1) $(13,15)$. Which of these theories is correct, or whether individual host factors interplay between one or more of these, is still unclear. However, the immune barrier hypothesis is the most inclusive for all of the components of all of these hypotheses as it involves impaired host defense, colonization by bacteria and fungi, loss of barrier function, an increased local innate and adaptive immune response, as well as local autoimmune response (15). Nasal polyps are a potential sequalae of this inflammatory reaction and occur in up to $30 \%$ of patients with CRS (16). Originally investigators believed this reaction was driven by type 2 inflammation, but recently this theory has been re-evaluated as the potential role of type 1 mediators in nasal polyps continues to expand (16). Treatment of each CRS subtype is typically aimed at the downstream inflammatory response, regardless of the underlying initiating pathophysiology (12).

\section{Classifying the Healthy Nasal Cavity Microbiota}

\section{Bacterial Environment}

The mucus membranes within the body provide an ideal environment for microbial nourishment (17). The characterization of bacteria within these environments can be accomplished with either culture-dependent or culture-independent analyses. Culture-dependent analyses rely on direct growth and identification of bacteria from a desired sample. Culture-dependent techniques are successful in identifying the most prominent bacterial colonizers, but many bacterial species are unable to grow outside of their natural body microhabitat and represents a limitation to this detection method $(18,19)$. Recently, advancements in culture-independent genetic sequencing and molecular analyses have resulted in the precise microbiologic characterization of the nasopharynx and sinus cavities. Typically, culture-independent techniques identify and amplify the 16s rRNA gene within a desired sample (19). The 16s rRNA gene is conserved within the bacterial genome, but each taxa demonstrates genetic variability within this region; thus, specific taxa can be identified from available genetic databases (20). Additionally, the 16s rRNA genome can be used to identify similar operational taxonomic units (OTUs) which are commonly used to quantify bacterial diversity (19).

The Human Microbiome Project (HMP), funded by the National Institutes of Health, was a foundational investigation which characterized the healthy bacterial microbiota and verified the presence of opportunistic pathogens within the anterior nares (21). Samples were collected from the anterior nares of 242 healthy participants which then underwent 16S rRNA gene profiling analysis. The opportunistic bacterium Staphylococcus aureus and Staphylococcus epidermidis were present in 29 and $93 \%$ of samples, respectively. Other notable opportunistic genera such as Propionibacterium, Corynebacterium, and Moraxella were highly abundant in select healthy anterior nares samples, although their prevalence varied. In contrast, the often-virulent pathogen Pseudomonas aeruginosa was absent in all anterior nare samples.

Subsequently, more recent studies have further examined the microbiota deeper within the nasal cavity. Bassis et al. 


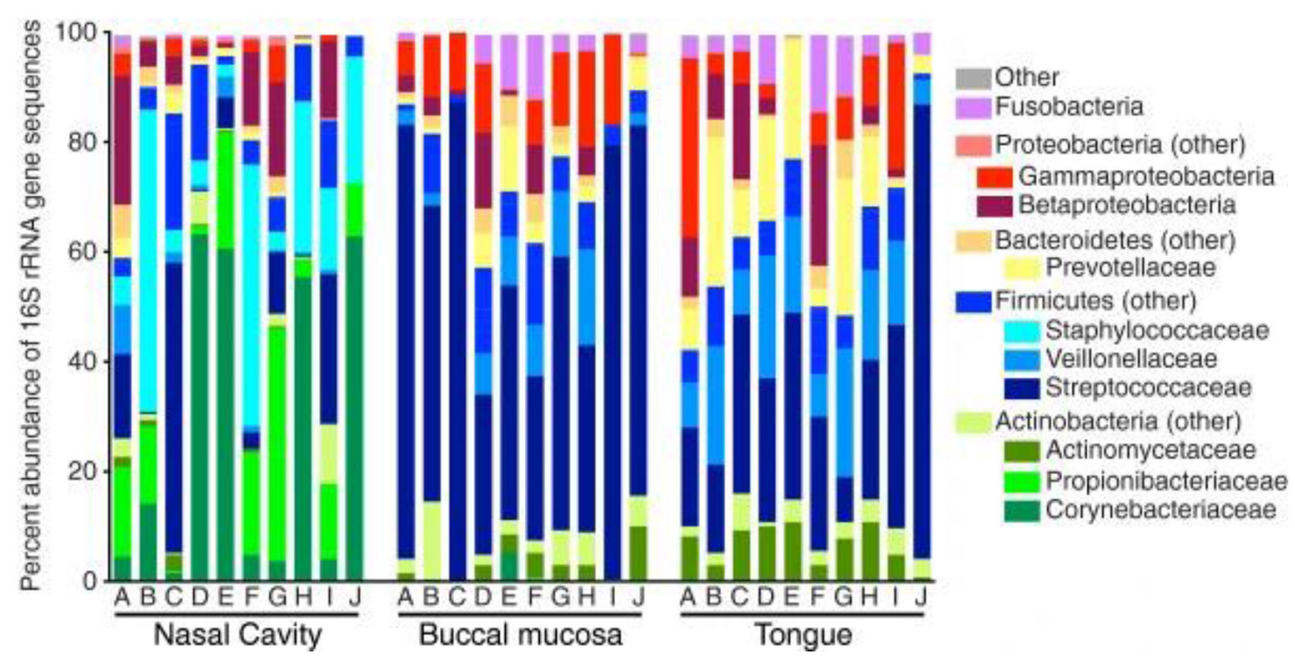

FIGURE 1 | Bacterial composition within the healthy nasal cavity, compared to the buccal mucosa and tongue. Data presented as relative abundances, and each column (marked by letters A through J) represents an independent study participant. Reprinted from (22), with permission under the Creative Commons Attribution License.

evaluated the middle meatus in healthy adults via cultureindependent 16S rRNA gene analysis with 269 sequences per sample (22). Comparable to the anterior nares from the HMP, Corynebacteria were highly abundant within the middle meatus, constituting at least half of the bacterial profile in $40 \%$ of the participants and were ubiquitous in the remaining subjects (Figure 1). Propionibacterium were also ubiquitous, ranging from 0.4 to $42.4 \%$ abundance. Staphylococcaceae genera were also present in all subjects, ranging from 2.2 to $55.0 \%$ abundance, although differentiation between the species aureus and epidermidis could not be determined. Notably, Moraxellacae genera were not widely prevalent, and were absent in $40 \%$ of participants. These genera have been noted to be more prevalent in children compared to adults (23). Other larger cultureindependent analyses have found comparable distributions in the middle meatus of healthy adults, with a high prevalence of Staphylococcaceae, Propionibacterium, and Corynebacterium as well as the detection of the opportunistic pathogens Streptococcus pneumoniae, Moraxella catarrhalis, and Haemophilus influenzae in select individuals (24).

\section{Emerging Fungal and Viral Nasal Microbiotas}

Fungi and viruses are noted components of the healthy human flora, but knowledge regarding their contributions toward the nasal microbiota is evolving (25-27). Within the anterior nares, the fungal species Malassezia predominates (28). Similarly, Malassezia is highly prevalent within the sinonasal cavity, with an average abundance of 53.9\% (29). Cladosporium (5.96\%) and Pleosporles $(2.17 \%)$ were the next most abundant fungal species, with the remaining community composed of a range of diverse species (29). Notably, Malassezia restrica abundance has an inverse relationship with Propionibacterium, which may imply a competing relationship. (29).
Symptomatic viral infections of the upper airway have been shown to alter the homeostatic bacterial community, but investigations into the viral microbiota (e.g., the virome), of asymptomatic and healthy individuals continues to be explored (30). Asymptomatic hosts may possess common viruses such as rhinovirus, enterovirus, and annellovirus within the respiratory tract microbiome (31). Bacteriophages have also been isolated within the anterior nares, including those that infect Propionibacterium (32). Phages of the human virome have demonstrated an immunomodulatory effect and can influence the composition of the bacterial microbiota (17). Overall, the literature on the healthy nasal virome and fungal contributions is limited and remains an important topic for future investigations. Table 1 compares the common bacterial, viral and fungal microbiota in healthy normal individuals to subjects with allergic rhinitis, CRSwNP and CRSsNP.

\section{COMPARING THE MICROBIOTAS OF CRSWNP AND CRSSNP}

The pathogenetic mechanisms of CRSwNP and CRSsNP are diverse, and thus it has been hypothesized that differing microbiotas may influence disease formation. Systematic reviews have further demonstrated that the nasal microbiota differs significantly in diseased states (19). Recently, Wei et al. compared the bacterial composition of the middle meatus in CRSwNP patients, CRSsNP patients, and patients without a diagnosis of CRS (13). In this culture-dependent investigation, the most commonly isolated strains from 136 cases of CRSwNP were Corynebacterium (19.9\%), S. epidermidis (19.1\%), Streptococcus (14.7\%), and S. aureus (11.0\%); the most prominently isolated strains in the 66 CRSsNP cases were Corynebacterium (21.2\%), S. epidermidis (21.2\%), S. aureus (13.6\%), and Streptococcus (7.6\%). Similarly, these four gram-positive bacteria composed 
TABLE 1 | Comparison Of Common Bacteria, Viruses And Fungi In Normal, Allergic Rhinitis, CRSwNP And CRSsNP.

\begin{tabular}{|c|c|c|c|}
\hline & Bacteria & Fungi & Viruses \\
\hline Healthy & $\begin{array}{l}\text { Propionibacterium spp. }(21,22,24,25) \\
\text { Corynebacterium spp. }(21,22,24,25) \\
\text { Moraxella spp. }(21,23) \\
\text { Staphylococcus spp. }(21,22,24,25) \\
\text { Streptococcus pneumoniae } \\
\text { Moraxella catarrhalis } \\
\text { Haemophilus influenza }\end{array}$ & $\begin{array}{l}\text { Malassezia (29) } \\
\text { Cladosporium (30) } \\
\text { Pleosporles (30) }\end{array}$ & $\begin{array}{l}\text { Rhinovirus (32) } \\
\text { Enterovirus (32) } \\
\text { Annelovirus (32) }\end{array}$ \\
\hline CRSsNP & $\begin{array}{l}\text { Corynebacterium spp. (13) } \\
\text { Staphylococcus spp. (13) } \\
\text { Streptococcus spp. (13) } \\
\text { Haemophilus influenzae (34) }\end{array}$ & & \\
\hline
\end{tabular}

the majority of isolated strains in the 17 control cases, and when compared across the three study cohorts, there was no statistically significant difference in abundance. The only strain identified as statistically distinct across the three study groups was Citrobacter, with a $5.9 \%$ isolation rate found in CRSwNP and no isolated strains found in the CRSsNP and control groups $(p=0.034)$. However, when the CRSwNP group was further stratified by eosinophil percentage in the peripheral blood, $S$. aureus was more common in the elevated eosinophil subgroup compared to the normal eosinophil percentage subgroup (17.2 vs. $3.3 \% ; p=0.011$ ). These findings are agreeable with previous studies which concluded an association between $S$. aureus in the middle meatus and CRS with allergic asthma (36). In contrast, Wei et al. found that $S$. epidermidis decreased as eosinophil percentages increased (13). Asthma was also associated with increased isolation rates of Corynebacterium and Pseudomonas within the CRSwNP population. There were no associated differences in bacteria isolation rates within the CRSsNP and control populations when controlled for eosinophilia or asthma. Fungal isolates were relatively uncommon and insignificant across cohorts (13).

The results from Wei et al. are comparable to other published reports investigating the nasal microbiota across the spectrum of CRS $(34,37)$. For example, Niederfuhr et al. concluded statistically insignificant differences in abundance of the most common bacterium, coagulase-negative Staphylococcus, Corynebacterium, and S. aureus, across CRS study groups (37). Although not statistically significant, the authors found S. aureus more commonly in CRSwNP compared to CRSsNP (23v. 8\%) and $H$. influenzae was more commonly detected in CRSsNP (31 v. $13 \%$ ) compared to CRSwNP (37). The authors from Niederfuhr et al. did not further stratify the study groups to investigate potential associations with eosinophilia, asthma, or other related characteristics. In contrast, Liu et al. stratified their CRSwNP cohort by peripheral eosinophil percentage and concluded an inverse association between eosinophilia and isolates of gram-negative aerobic and facultative anaerobic bacteria (34).

These microbiologic analyses provide insight into the potential associations between microbiota and CRS development. Although the most common bacteria were proportionally similar across CRS cohorts in these culture-dependent investigations, stratification by blood eosinophil level and asthma identified potential associations with CRSwNP and certain pathogens, specifically $S$. aureus and S. epidermidis. This stratification permits us to view CRS as a more continual spectrum of disease, rather than a binary process, and spurs hypotheses regarding the role of specific microbiota on immune modulation and pathogenesis. Furthermore, multivariate correspondence analyses demonstrate a potential positive association between CRSwNP and S. aureus, Streptococcus, Haemophilus, Enterobacter, and Corynebacterium, which was not apparent on observational chi square analyses (34). Similarly, culture-independent genetic analyses assert a greater abundance of $S$. aureus within CRSwNP patients compared to CRSsNP, which has influenced recent hypotheses explaining CRSwNP pathogenesis (33).

\section{Microbiota of Allergic Rhinitis}

Whereas CRS is generally associated with decreased microbiologic diversity, the available literature demonstrates AR may instead be associated with increased bacterial diversity relative to NAR and CRS cohorts $(8,38)$. In a study by Choi et al., the authors collected samples from the middle meatus and vestibule of 19 seasonal AR patients and 20 NAR patients. Outside of the allergy season, each cohort demonstrated comparable numbers of unique bacterial phyla $(p<0.14)$; however, during the symptomatic allergy period, the AR cohort reported significantly increased unique bacterial phyla compared 
to the NAR cohort $(p<0.036)$, although the authors did not report which specific bacterium differed. Further, the authors demonstrated a positive correlation between unique bacterial phyla in the middle meatus and nasal eosinophil count within the AR cohort during the allergy season $(\rho=0.35$ and $p<0.033)$, suggesting increased Th2 inflammation with bacterial diversity. Lal et al. compared bacterial species diversity within the middle meatus across CRSwNP, CRSsNP, and AR patients; their findings demonstrated that while AR and CRSwNP patients exhibited similar levels of bacterial diversity, each group was associated with higher number of unique bacterial phyla compared to CRSsNP $(p<0.05)$ (39). Gan et al. further identified increased Spirochaete abundance within AR nasal cavities compared to CRS patients, while the AR cohort also exhibited higher abundances of Pseudomonas and Peptostreptococcaceae than in control cohorts $(p<0.005)(40)$. However, in contrast to Choi et al., the authors did not collect AR samples during times of increased allergen exposure, which may represent a limitation to making direct comparisons between studies.

\section{Role of the Nasopharyngeal Microbiome in CRS and Polyp Development \\ Bacteriologic Influences}

CRS, by its definition, is driven by chronic inflammation at the mucosal surface. The nasal microbiota may contribute toward the pathogenesis of CRS and influence the inflammatory mechanism between Th1 or Th2 predominance (Figure 2). S. aureus has been one of the most extensively studied promoters of this underlying reaction $(35,36)$. Multiple hypotheses have attempted to explain this relationship, including a superantigen-induced local T-lymphocyte reaction with resultant IgE production (41). In this proposed mechanism, $S$. aureus releases superantigens which induces a potent local T-cell reaction. This cascade leads to production of Th2 cytokines such as IL-4, IL-5, and IL-13. The subsequent eosinophilia and B-cell activation results in specific IgE production against the $S$. aureus superantigens, further perpetuating the chronic Th2 inflammatory reaction $(42,43)$. Asthma, a common comorbidity in CRS patients, has also been associated with increased formation of specific IgE directed against the $S$. aureus superantigen (44).

In contrast, other experimental models have associated S. aureus with Th1 and Th17 inflammation, which is classically described in CRSsNP, further complicating the definitive role this bacterium may play in CRS development $(35,45)$. In this mechanism, $S$. aureus releases extracellular membrane vesicles (EV) which contain receptors, lipids, and nucleic acids, among other bioactive compounds $(35,46)$. These EVs induce subsequent Th1 and Th17 cytokine responses, leading to a predominately neutrophilic inflammatory reaction; in addition, S. aureus EVs do not cause augmentation of IL-4, a potent Th2-cytokine. Other $S$. aureus virulence factors such as surface protein A and lipoteichoic acid (LTA) have similarly been shown to induce histamine release and promote epithelial colonization, respectively $(42,47)$. Therefore, it is likely $S$. aureus plays a pathologic role in both CRS phenotypes and thus requires further investigation into whether it is functioning as a potential facilitator for both Th1 and Th2 chronic inflammation. These observations also promote the hypothesis that the CRS T-lymphocyte cascade occurs along a spectrum, rather than a strict binary Th1 or Th2 fashion as classically described $(48,49)$.

Alterations in nasal bacterial diversity have also been reported, which may also contribute toward CRS pathogenesis (50). Culture-based approaches, such as those discussed previously in the context of CRS bacterial abundance, are adequate for isolating prominent species but lack in their ability to estimate total microbiologic diversity $(9,51)$. Thus, genetic sequencing has been utilized to quantitatively assess these differences. Using 16s rRNA sampling, Abreu et al. concluded similar bacterial burdens within the maxillary sinuses of CRS patients and healthy controls (2.10 $\times 10^{6}$ vs. $2.92 \times 10^{6}$ rRNA gene copies per microgram; $p=0.37$ ), but found a significant decrease in species diversity within the CRS cohort ( $\sim 1,200$ vs. $\sim 950$ unique bacterial taxa in healthy and CRS patients, respectively; $p<0.008)(50)$. It is necessary to note some participants in this investigation had a history of antibiotic use, and although the authors minimize the role of this potential confounder on the results, previous studies have implicated antibiotic therapy with decreased bacterial diversity and an increased S. aureus abundance (36). In a study conducted by Chalermwatanachai et al. where antibiotic use was an exclusion criteria, the authors nevertheless concluded a significant decrease in bacterial diversity in CRSwNP patients compared to healthy controls; CRSsNP patients were not included in this analysis (52). In metagenomic investigations directly comparing bacterial diversity between CRSwNP and CRSsNP patients, the nasal cavity of the CRSsNP cohort demonstrated significantly decreased bacterial species diversity, and each CRS phenotype (wNP and sNP) possessed significantly fewer unique bacterial species compared to healthy controls (33).

The precise influence of species diversity on CRS development remains under investigation. Proposed mechanisms include a loss of "protective" bacteria and the formation of biofilms. One bacteria hypothesized to serve a protective role is Propionibacterium acnes (52). Laboratory models demonstrate $P$. acnes releases propionic acid, which attenuates macrophageinduced inflammation and decreases growth of S. aureus (53, 54). One genetic investigation concluded $P$. acnes is more common in healthy individuals compared to CRSwNP, but the detected levels of propionic acid were not high enough to exert these protective effects in vivo (52). Nevertheless, $P$. acnes remains a bacterium of interest as well as others including Lactobacillus sakei, Dolosigranulum genera, and Citrobacter genera. $(13,50,55)$ Lactobacillus species are capable of producing lactic acid which may outcompete pathogenic species (50). Dolosigranulum genera have been associated with decreased rates of respiratory infections and abundance is inversely related to $S$. aureus colonization (55). Citrobacter is a common intestinal bacterium that is also noted to be significantly more abundant within the nasal cavity of CRSwNP patients compared to CRSsNP, spurring potential investigations into potential immune-protective properties (13). Bacterial dysbiosis, such as reductions in bacterial diversity with concomitant abundances of S. aureus and Pseudomonas, are also hypothesized to influence 


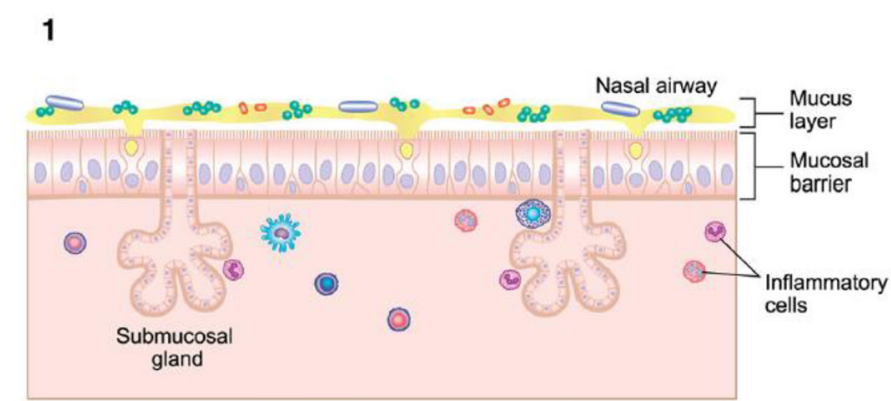

Normal nasal mucosa and colonization with microbes

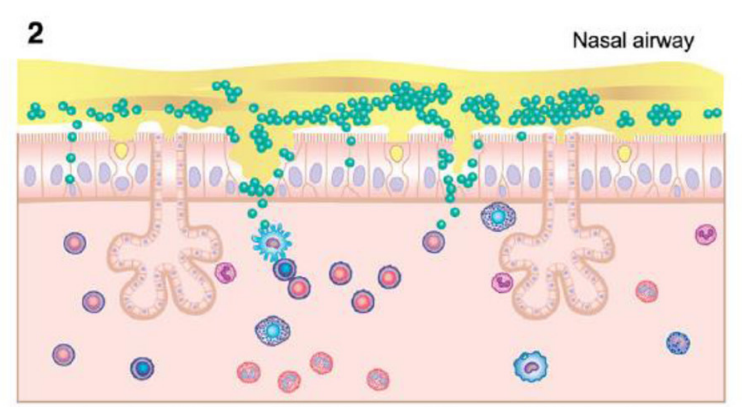

Loss of barrier with increased abundance and decreased diversity of microbes

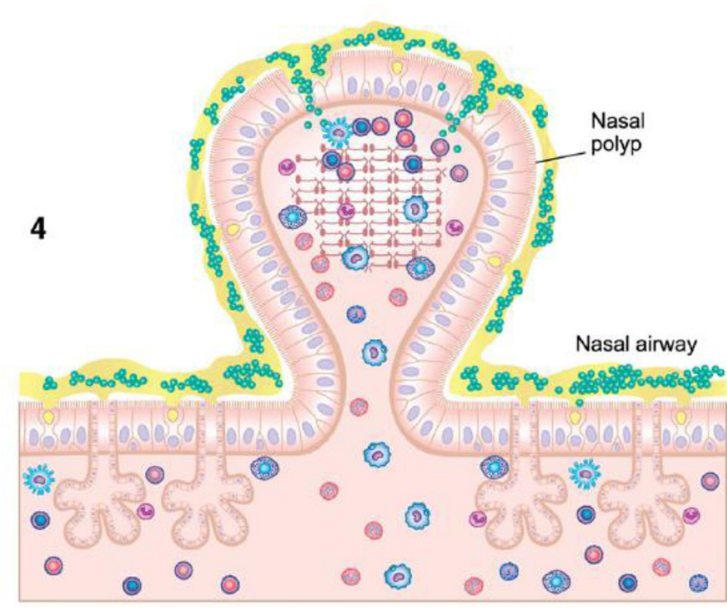

Tissue remodeling with loss of submucosal glands in polyp and profound inflammatory cell expansion
Recruitment and expansion of inflammatory cells, tissue swelling,

inflammation, and deposition of crosslinked fibrin

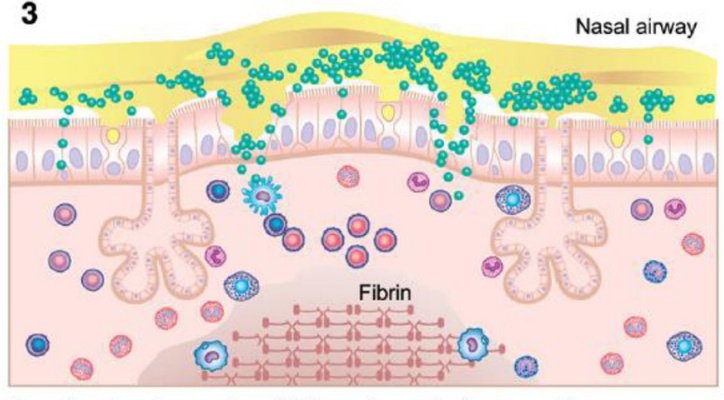

FIGURE 2 | CRSWNP immunopathogenesis (13) - colonization of microbes with accumulation of immune cells can lead to tissue injury, inflammation, and mucosal barrier loss in CRS.

the dynamics of nasal biofilms $(56,57)$. These biofilms confer a high resistance to antimicrobial defenses and can result in longstanding inflammation at the mucosal surface (58). Although present in both healthy and CRS patients, their presence had been associated with poorer treatment outcomes (59).

\section{Viral Influences}

Respiratory viruses such as rhinoviruses, coronaviruses, and influenza viruses are routinely found within the nasal cavity of CRS patients, but relatively few studies have examined these viral associations between CRS phenotypes $(60,61)$. In one study conducted by Goggin et al., the authors demonstrated the aforementioned respiratory viruses are associated more with CRSsNP populations, compared to CRSwNP and healthy controls (62). However, the authors concluded no difference in disease severity between CRS phenotypes. Multiple proposed mechanisms for viral influence on CRS development exist and further investigations are warranted. Current evidence implies that replicating respiratory viruses may damage epithelial barriers, which facilitates environmental exposure and perpetuates inflammation $(63,64)$. Other proposed mechanisms include an impairment of ciliary function and mucus overproduction $(65,66)$. It remains unclear if viral influences perpetuate chronic inflammation within CRS or contribute to the initial disease stimulus (67).

\section{Modifying Disease Risk}

Current research supports that a reduction in the proportion of potentially protective strains of bacteria may decrease the stability of the sinunasal microbiome and ultimately contribute to disease progression, severity, and CRS phenotype $(68,69)$. Therefore, modifying certain risk factors and targeted treatments may shape tailored therapy in patients with CRS (70). For example, literature has supported the use of probiotic applications to decrease the density of certain bacteria, including pathogenic strains of Staphylococcus aureus, associated in high concentrations with the microbiome of CRS (71-73). These effects have also been seen with the use of intranasal probiotic irrigation (74). Furthermore, evidence suggests that certain probiotic strains have the potential to have immunomodulatory effects that favor the stimulation of a Th1 profile, further augmenting humoral immunity with increases in IgA production and enhanced mucosal defense immune responses (75). Another potential method to modify disease risk by further altering the nasal microbiome is the use of intranasal corticosteroids; the use of intranasal corticosteroids has been shown to modify the commensal microbiota and may 
further serve to decrease the density of pathogenic strains of colonizing bacteria related to the development of CRS (76).

CRS pathogenesis also involves the increased presence of local inflammatory mediators due to mucosal irritation (77). The risk for CRS may be reduced by increasing the consumption of anti-inflammatory foods and decreasing the frequency of meat and fat intake (78). Tobacco smoke, a local irritant to the nasal mucosa, is thought to be involved in the pathogenesis and symptom burden of CRS (79). For example, patient smoking history has been determined to have a substantial influence on sinunasal bacterial colonization and modification of nasal mucosal physiological and immunological properties (80).

\section{LIMITATIONS}

As previously mentioned, our understanding of the microbiologic influences on CRS is rapidly expanding but still incompletely understood (1). Thus, it is challenging for a single review to comprehensively capture all recent developments and perspectives. Additionally, as demonstrated by multiple systemic analyses, there is notable variation in the microbiologic landscape between research studies $(19,81)$. Such variation is present across both healthy and CRS subjects, indicating lack of generalizability of these studies as the microbiome is likely affected by a spectrum of environmental and host determinants; factors such as these may complicate participant selection in large-scale investigations $(82,83)$. In addition, a lack of conformity in methods for collection of specimens, culture techniques and in DNA/RNA extraction can contribute to the variability of results reported $(84,85)$. Other factors such as antibiotic use, disease severity, and patient age must also be controlled for when investigating the microbiome (86).

\section{CONCLUSIONS}

CRS is a clinical syndrome which has a broad scope of clinical presentations and treatment response. It is clear that CRSwNP and CRSsNP represent two distinct phenotypes and that CRSwNP is likely driven by Type 2 inflammation; yet questions still remain on how the nasal cavity microbiome influences these divergent clinical conditions.

Treatment of CRS is currently aimed at reducing inflammation and obstructive defects by either medical or surgical modalities. Current medical therapy for both CRS phenotypes may include nasal saline irrigation and intranasal corticosteroids, while operative therapies such as functional endoscopic sinus surgery may help restore sinus ventilation (8789). However, recent approval by regulatory agencies for biologic therapies that target Type 2 inflammation including dupilumab (an anti-IL-4 $\alpha$ monoclonal antibody) (90), omalizumab (an anti-IgE monoclonal antibody), and mepolizumab (anti-IL-5) have been found to be efficacious at reducing nasal polyps in patients with CRSwNP. This has resulted in controversial treatment paradigm shifts regarding when to use these agents.
Should they be used in lieu of surgery or be reserved for use post-operatively in order to achieve the maximum desired effect? (91) Further well-controlled studies will be needed to answer this question that will also need to factor in economic costs of surgery and biologic use.

nProbiotics may play an important treatment role in CRS via modification of the GI-respiratory axis resulting in attenuation of underlying inflammation $(75,92)$ as they can alter the host-microbiome composition and immune function, including the production of antimicrobial peptides and compounds (72). In addition, alteration of the host-microbiome composition can result in a microbial-driven shift in development of immunomodulatory cells and cytokines such as Foxp3+ $\mathrm{T}$ reg cells and IL-10, respectively (72). However, studies that have used probiotics to modify clinical symptoms in CRS and other airways diseases have been mixed, with most unable to produce a detectable long-term improvement in clinical symptoms $(93,94)$.

Rhinitis may present similarly to CRS, and investigations comparing their respective microbiologic environments are ongoing. The current literature implies AR may be associated with increased bacterial species diversity compared to NAR and CRS (38). Like CRS, AR treatments are aimed at attenuating the underlying Type 2 inflammation using intranasal corticosteroids and antihistamines (95). As discussed, biologics are effective in patients with CRSwNP and other comorbid allergic conditions such as asthma but less is known how they might affect the nasal microbiome in $\mathrm{AR}$ or other chronic rhinitis subtypes (96). Allergen immunotherapy (AIT) is another effective treatment modality for AR (97). However, studies investigating changes to the underlying microbiota during AIT have been unable to correlate symptomatic improvement with detectable changes in nasal microbial diversity (38). Interestingly, the nasal microbiota following symptomatic improvement with AIT does not resemble the microbiota of a non-atopic healthy individual (38).

In summary, this review is not intended to be an exhaustive assessment of all research pertaining to CRS and the microbiome, but rather to illustrate the current understanding and gaps in knowledge pertaining to this important area of research. As with many chronic inflammatory diseases, the underlying pathophysiology of CRS and chronic rhinitis is a complex interplay between genetics, barrier function, immune function and the resident microbiome composition and balance. Targeted approaches that can favorably alter the pathogenic microbiome will likely become an important part of a multifaceted treatment approach for CRS.

\section{AUTHOR CONTRIBUTIONS}

$\mathrm{KH}$, JR, and LF contributed toward the writing of the manuscript. $\mathrm{KH}$ led the design and implementation of the manuscript outline. $\mathrm{KH}, \mathrm{JR}, \mathrm{LF}$, and JB provided research and literature necessary for the review. JB provided final supervision and ensured the accuracy of all texts. 


\section{REFERENCES}

1. Young VB. The role of the microbiome in human health and disease: an introduction for clinicians. BMJ. (2017) 356:j831. doi: 10.1136/bmj.j831

2. Marchesi JR, Ravel J. The vocabulary of microbiome research: a proposal. Microbiome. (2015) 3:31. doi: 10.1186/s40168-015-0094-5

3. Cockburn DW, Koropatkin NM. Polysaccharide degradation by the intestinal microbiota and its influence on human health and disease. J Mol Biol. (2016) 428:3230-3252. doi: 10.1016/j.jmb.2016.06.021

4. Casadevall A, Pirofski LA. What is a host? Incorporating the microbiota into the damage-response framework. Infect Immunity. (2015) 83:27. doi: 10.1128/IAI.02627-14

5. He Y, Wen Q, Yao F, Xu D, Huang Y, Wang J. Gut-lung axis: the microbial contributions and clinical implications. Crit Rev Microbiol. (2017) 43:8195. doi: 10.1080/1040841X.2016.1176988

6. Segal LN, Blaser MJ. A brave new world: the lung microbiota in an era of change. Ann Am Thor Soc. (2014) 11(Suppl 1):S21-27. doi: 10.1513/AnnalsATS.201306-189MG

7. Watts AM, West NP, Zhang P, Smith PK, Cripps AW, Cox AJ. The gut microbiome of adults with allergic rhinitis is characterised by reduced diversity and an altered abundance of key microbial taxa compared to controls. Int Arch Allergy Immunol. (2021) 182:94-105. doi: 10.1159/000510536

8. Mazmanian SK, Liu CH, Tzianabos AO, Kasper DL. An immunomodulatory molecule of symbiotic bacteria directs maturation of the host immune system. Cell. (2005) 122:107-18. doi: 10.1016/j.cell.2005.05.007

9. Wilson MT, Hamilos DL. The nasal and sinus microbiome in health and disease. Curr Allergy Asthma Rep. (2014) 14:485. doi: 10.1007/s11882-014-0485-x

10. Skoner DP. Allergic rhinitis: definition, epidemiology, pathophysiology, detection, and diagnosis. J Allergy Clin Immunol. (2001) 108:S28. doi: 10.1067/mai.2001.115569

11. Meltzer EO, Hamilos DL, Hadley JA, Lanza DC, Marple BF, Nicklas RA, et al. Rhinosinusitis: establishing definitions for clinical research and patient care. J Allergy Clin Immunol. (2004) 114(Suppl. 6):155212. doi: 10.1016/j.jaci.2004.09.029

12. Sedaghat AR. Chronic rhinosinusitis. In: American Family Physician. vol 96 (2017). p. 500-6.

13. Wei H-Z, Li Y-C, Wang X-D, Lu X-X, Hu C-H, He S, et al. The microbiology of chronic rhinosinusitis with and without nasal polyps. Eur Arch Oto Rhino Laryngol. (2018) 275:1439-47. doi: 10.1007/s00405-018-4931-6

14. Bachert C, Pawankar R, Zhang L, Bunnag C, Fokkens WJ, Hamilos DL, et al. ICON: chronic rhinosinusitis. World Allergy Organi J. (2014) 7:25. doi: 10.1186/1939-4551-7-25

15. Lam K, Schleimer R, Kern RC. The etiology and pathogenesis of chronic rhinosinusitis: a review of current hypotheses. Curr Allergy Asthma Rep. (2015) 15:41. doi: 10.1007/s11882-015-0540-2

16. Stevens WW, Schleimer RP, Kern RC. Chronic rhinosinusitis with nasal polyps. J Allergy Clin Immunol Pract. (2016) 4:56572. doi: 10.1016/j.jaip.2016.04.012

17. Barr JJ. A bacteriophages journey through the human body. Immunol Rev. (2017) 279:106-22. doi: 10.1111/imr.12565

18. Dickson RP, Erb-Downward JR, Prescott HC, Martinez FJ, Curtis JL, Lama VN, et al. Analysis of culture-dependent versus culture-independent techniques for identification of bacteria in clinically obtained bronchoalveolar lavage fluid. J Clin Microbiol. (2014) 52:3605-13. doi: 10.1128/JCM.01028-14

19. Lee JT, Frank DN, Ramakrishnan V. Microbiome of the paranasal sinuses: update and literature review. Am J Rhinol Allergy. (2016) 30:316. doi: 10.2500/ajra.2016.30.4255

20. Schütte UM, Abdo Z, Bent SJ, Shyu C, Williams CJ, Pierson JD, et al. Advances in the use of terminal restriction fragment length polymorphism (T-RFLP) analysis of $16 \mathrm{~S}$ rRNA genes to characterize microbial communities. Appl Microbiol Biotechnol. (2008) 80:365-80. doi: 10.1007/s00253-008-1565-4

21. Huttenhower C, Gevers D, Knight R, Abubucker S, Badger JH, Chinwalla AT, et al. Structure, function and diversity of the healthy human microbiome. Nature. (2012) 486:207-14. doi: 10.1038/nature11234

22. Bassis CM, Tang AL, Young VB, Pynnonen MA. The nasal cavity microbiota of healthy adults. Microbiome. (2014) 2:27. doi: 10.1186/2049-2618-2-27
23. Stearns JC, Davidson CJ, McKeon S, Whelan FJ, Fontes ME, Schryvers $\mathrm{AB}$, et al. Culture and molecular-based profiles show shifts in bacterial communities of the upper respiratory tract that occur with age. ISME J. (2015) 9:1246-59. doi: 10.1038/ismej.2014.250

24. Ramakrishnan VR, Feazel LM, Gitomer SA, Ir D, Robertson CE, Frank DN. The microbiome of the middle meatus in healthy adults. PLoS ONE. (2013) 8:e85507. doi: 10.1371/journal.pone.0085507

25. Liang G, Bushman FD. The human virome: assembly, composition and host interactions. Nat Rev Microbiol. (2021) 2021:1-14 doi: 10.1038/s41579-021-00536-5

26. Blum HE. The human microbiome. Adv Med Sci. (2017) 62:41420. doi: 10.1016/j.advms.2017.04.005

27. Kumpitsch C, Koskinen K, Schöpf V, Moissl-Eichinger C. The microbiome of the upper respiratory tract in health and disease. BMC Biol. (2019) 17:87. doi: 10.1186/s12915-019-0703-z

28. Findley K, Oh J, Yang J, Conlan S, Deming C, Meyer JA, et al. Topographic diversity of fungal and bacterial communities in human skin. Nature. (2013) 498:367-70. doi: 10.1038/nature12171

29. Wagner Mackenzie B, Chang K, Zoing M, Jain R, Hoggard M, Biswas K, et al. Longitudinal study of the bacterial and fungal microbiota in the human sinuses reveals seasonal and annual changes in diversity. Sci Rep. (2019) 9:17416. doi: 10.1038/s41598-019-53975-9

30. Bosch AA, Biesbroek G, Trzcinski K, Sanders EA, Bogaert D. Viral and bacterial interactions in the upper respiratory tract. PLoS Patho. (2013) 9:e1003057. doi: 10.1371/journal.ppat.1003057

31. Wylie KM. The virome of the human respiratory tract. Clin Chest Med. (2017) 38:11-9. doi: 10.1016/j.ccm.2016.11.001

32. Lloyd-Price J, Mahurkar A, Rahnavard G, Crabtree J, Orvis J, Hall AB, et al. Strains, functions and dynamics in the expanded human microbi32ome project. Nature. (2017) 550:61-6. doi: 10.1038/nature23889

33. Choi E-B, Hong S-W, Kim D-K, Jeon SG, Kim K-R, Cho S-H, et al. Decreased diversity of nasal microbiota and their secreted extracellular vesicles in patients with chronic rhinosinusitis based on a metagenomic analysis. Allergy. (2014) 69:517-26. doi: 10.1111/all.12374

34. Liu Q, Lu X, Bo M, Qing H, Wang X, Zhang L. The microbiology of chronic rhinosinusitis with and without nasal polyps. Acta Oto Laryngol. (2014) 134:1251-8. doi: 10.3109/00016489.2013.879737

35. Kim M-R, Hong S-W, Choi E-B, Lee W-H, Kim Y-S, Jeon SG, et al. Staphylococcus aureus-derived extracellular vesicles induce neutrophilic pulmonary inflammation via both Th1 and Th17 cell responses. Allergy. (2012) 67:1271-81. doi: 10.1111/all.12001

36. Feazel LM, Robertson CE, Ramakrishnan VR, Frank DN. Microbiome complexity and staphylococcus aureus in chronic rhinosinusitis. Laryngoscope. (2012) 122:467-72. doi: 10.1002/lary.22398

37. Niederfuhr A, Kirsche H, Riechelmann H, Wellinghausen N. The bacteriology of chronic rhinosinusitis with and without nasal polyps. Arch Otolaryngol Head Neck Surg. (2009) 135:131-6. doi: 10.1001/archoto.2008.531

38. Bender ME, Read TD, Edwards TS, Hargita M, Cutler AJ, Wissel EF, et al. A comparison of the bacterial nasal microbiome in allergic rhinitis patients before and after immunotherapy. Laryngoscope. (2020) 130:E882c8. doi: 10.1002/lary.28599

39. Lal D, Keim P, Delisle J, Barker B, Rank MA, Chia N, et al. Mapping and comparing bacterial microbiota in the sinonasal cavity of healthy, allergic rhinitis, and chronic rhinosinusitis subjects. Int Forum Allergy Rhinol. (2017) 7:561-9. doi: 10.1002/alr.21934

40. Gan W, Yang F, Meng J, Liu F, Liu S, Xian J. Comparing the nasal bacterial microbiome diversity of allergic rhinitis, chronic rhinosinusitis and control subjects. Eur Arch Oto Rhino Laryngol. (2021) 278:7118. doi: 10.1007/s00405-020-06311-1

41. Hamilos DL. Drivers of chronic rhinosinusitis: inflammation versus infection. J Allergy Clin Immunol. (2015) 136:1454-9. doi: 10.1016/j.jaci.2015.10.011

42. Patou J, Gevaert P, Van Zele T, Holtappels G, van Cauwenberge P, Bachert C. Staphylococcus aureus enterotoxin B, protein A, and lipoteichoic acid stimulations in nasal polyps. J Allergy Clin Immunol. (2008) 121:1105. doi: 10.1016/j.jaci.2007.08.059

43. Tantilipikorn P, Bunnag C, Nan Z, Bachert C. Staphylococcus aureus superantigens and their role in eosinophilic nasal polyp disease. Asian Pacific J Allergy Immunol. (2012) 30:171-6. 
44. Bachert C, Zhang N, Holtappels G, De Lobel L, van Cauwenberge P, Liu S, et al. Presence of IL-5 protein and IgE antibodies to staphylococcal enterotoxins in nasal polyps is associated with comorbid asthma. J Allergy Clin Immunol. (2010) 126:962-8.e966. doi: 10.1016/j.jaci.2010.07.007

45. Van Bruaene N, Pérez-Novo CA, Basinski TM, Van Zele T, Holtappels $\mathrm{G}$, De Ruyck $\mathrm{N}$, et al. T-cell regulation in chronic paranasal sinus disease. J Allergy Clin Immunol. (2008) 121:1435-41:1441.e14313. doi: 10.1016/j.jaci.2008.02.018

46. Tetta C, Ghigo E, Silengo L, Deregibus MC, Camussi G. Extracellular vesicles as an emerging mechanism of cell-to-cell communication. Endocrine. (2013) 44:11-9. doi: 10.1007/s12020-012-9839-0

47. Weidenmaier C, Kokai-Kun JF, Kristian SA, Chanturiya T, Kalbacher H, Gross $\mathrm{M}$, et al. Role of teichoic acids in staphylococcus aureus nasal colonization, a major risk factor in nosocomial infections. Nat Med. (2004) 10:2435. doi: $10.1038 / \mathrm{nm} 991$

48. Derycke L, Eyerich S, Van Crombruggen K, Pérez-Novo C, Holtappels G, Deruyck N, et al. Mixed T helper cell signatures in chronic rhinosinusitis with and without polyps. PLoS ONE. (2014) 9:e97581. doi: 10.1371/journal.pone.0097581

49. Cao PP, Wang ZC, Schleimer RP, Liu Z. Pathophysiologic mechanisms of chronic rhinosinusitis and their roles in emerging disease endotypes. Ann Allergy Asthma Immunol. (2019) 122:33-40. doi: 10.1016/j.anai.2018.10.014

50. Abreu NA, Nagalingam NA, Song Y, Roediger FC, Pletcher SD, Goldberg AN, et al. Sinus microbiome diversity depletion and $<\mathrm{em}>$ Corynebacterium tuberculostearicum $</ \mathrm{em}>$ enrichment mediates rhinosinusitis. Sci Transl Med. (2012) 4:151 ra124. doi: 10.1126/scitranslmed.3003783

51. Hauser LJ, Feazel LM, Ir D, Fang R, Wagner BD, Robertson CE, et al. Sinus culture poorly predicts resident microbiota. Int Forum Allergy Rhinol. (2015) 5:3-9. doi: 10.1002/alr.21428

52. Chalermwatanachai T, Vilchez-Vargas R, Holtappels G, Lacoere T, Jáuregui $\mathrm{R}$, Kerckhof F-M, et al. Chronic rhinosinusitis with nasal polyps is characterized by dysbacteriosis of the nasal microbiota. Sci Rep. (2018) 8:7926. doi: 10.1038/s41598-018-26327-2

53. Wang Y, Dai A, Huang S, Kuo S, Shu M, Tapia CP, et al. Propionic acid and its esterified derivative suppress the growth of methicillinresistant staphylococcus aureus USA300. Benefic Micro. (2014) 5:1618. doi: 10.3920/BM2013.0031

54. Al-Lahham S, Roelofsen H, Rezaee F, Weening D, Hoek A, Vonk $\mathrm{R}$, et al. Propionic acid affects immune status and metabolism in adipose tissue from overweight subjects. Eur J Clin Inv. (2012) 42:35764. doi: 10.1111/j.1365-2362.2011.02590.x

55. Hasegawa K, Linnemann RW, Mansbach JM, Ajami NJ, Espinola JA, Petrosino JF, et al. Nasal airway microbiota profile and severe bronchiolitis in infants: a case-control study. Pediatr Infect Dis J. (2017) 36:104451. doi: 10.1097/INF.0000000000001500

56. Hoggard M, Wagner Mackenzie B, Jain R, Taylor MW, Biswas K, Douglas RG. Chronic rhinosinusitis and the evolving understanding of microbial ecology in chronic inflammatory mucosal disease. Clin Microbiol Rev. (2017) 30:321-48. doi: 10.1128/CMR.00060-16

57. Lee K, Pletcher SD, Lynch SV, Goldberg AN, Cope EK. Heterogeneity of microbiota dysbiosis in chronic rhinosinusitis: potential clinical implications and microbial community mechanisms contributing to sinonasal inflammation. Front Cell Infect Microbiol. (2018) 8. doi: $10.3389 /$ fcimb. 2018.00168

58. Fastenberg JH, Hsueh WD, Mustafa A, Akbar NA, Abuzeid WM. Biofilms in chronic rhinosinusitis: pathophysiology and therapeutic strategies. World J Otorhinolaryngol Head Neck Surg. (2016) 2:219-29. doi: 10.1016/j.wjorl.2016.03.002

59. Bendouah Z, Barbeau J, Hamad WA, Desrosiers M. Biofilm formation by staphylococcus aureus and pseudomonas aeruginosa is associated with an unfavorable evolution after surgery for chronic sinusitis and nasal polyposis. Otolaryngol Head Neck Surgery. (2006) 134:991-6. doi: 10.1016/j.otohns.2006.03.001

60. Liao B, Hu C-Y, Liu T, Liu Z. Respiratory viral infection in the chronic persistent phase of chronic rhinosinusitis. Laryngoscope. (2014) 124:8327. doi: 10.1002/lary.24348

61. Cho GS, Moon B-J, Lee B-J, Gong C-H, Kim NH, Kim Y-S, et al. High rates of detection of respiratory viruses in the nasal washes and mucosae of patients with chronic rhinosinusitis. J Clin Microbiol. (2013) 51:97984. doi: 10.1128/JCM.02806-12

62. Goggin RK, Bennett CA, Bialasiewicz S, Vediappan RS, Vreugde S, Wormald P-J, et al. The presence of virus significantly associates with chronic rhinosinusitis disease severity. Allergy. (2019) 74:1569-72. doi: 10.1111/all.13772

63. Yeo NK, Jang YJ. Rhinovirus infection-induced alteration of tight junction and adherens junction components in human nasal epithelial cells. Laryngoscope. (2010) 120:346-52. doi: 10.1002/lary.20764

64. Sivasubramaniam R, Douglas R. The microbiome and chronic rhinosinusitis. World J Otorhinolaryngol Head Neck Surg. (2018) 4:216-21. doi: 10.1016/j.wjorl.2018.08.004

65. Jackson DJ, Johnston SL. The role of viruses in acute exacerbations of asthma. J Allergy Clin Immunol. (2010) 125:1178-87; quiz 11881179. doi: $10.1016 /$ j.jaci.2010.04.021

66. He SH, Zheng J, Duan MK. Induction of mucin secretion from human bronchial tissue and epithelial cells by rhinovirus and lipopolysaccharide. Acta pharmacol Sinica. (2004) 25:1176-81.

67. Wood AJ, Antoszewska H, Fraser J, Douglas RG. Is chronic rhinosinusitis caused by persistent respiratory virus infection? Int Forum Allergy Rhinol. (2011) 1:95-100. doi: 10.1002/alr.20030

68. Gan W, Yang F, Tang Y, Zhou D, Qing D, Hu J, et al. The difference in nasal bacterial microbiome diversity between chronic rhinosinusitis patients with polyps and a control population. Int Forum Allergy Rhinol. (2019) 9:582-92. doi: 10.1002/alr.22297

69. Dimitri-Pinheiro S, Soares R, Barata P. The microbiome of the nose-friend or foe? Allergy Rhinol. (2020) 11:2152656720911605. doi: 10.1177/2152656720911605

70. Cope EK, Goldberg AN, Pletcher SD, Lynch SV. Compositionally and functionally distinct sinus microbiota in chronic rhinosinusitis patients have immunological and clinically divergent consequences. Microbiome. (2017) 5:53. doi: 10.1186/s40168-017-0266-6

71. Cleland EJ, Drilling A, Bassiouni A, James C, Vreugde S, Wormald P-J. Probiotic manipulation of the chronic rhinosinusitis microbiome. Int Forum Allergy Rhinol. (2014) 4:309-14. doi: 10.1002/alr.21279

72. Cope EK, Lynch SV. Novel microbiome-based therapeutics for chronic rhinosinusitis. Curr Allergy Asthma Rep. (2015) 15:9. doi: 10.1007/s11882-014-0504-y

73. Jain R, Hoggard M, Zoing M, Jiang Y, Biswas K, Taylor MW, et al. The effect of medical treatments on the bacterial microbiome in patients with chronic rhinosinusitis: a pilot study. Int Forum Allergy Rhinol. (2018) 8:8909. doi: 10.1002/alr.22110

74. Endam LM, Alromaih S, Gonzalez E, Madrenas J, Cousineau B, Renteria $\mathrm{AE}$, et al. Intranasal application of lactococcus lactis W136 is safe in chronic rhinosinusitis patients with previous sinus surgery. Front Cell Infect Microbiol. (2020) 10:1-15. doi: 10.3389/fcimb.2020.00440

75. Kramer MF, Heath MD. Probiotics in the treatment of chronic rhinoconjunctivitis and chronic rhinosinusitis. J Allergy. (2014) 2014:983635. doi: 10.1155/2014/983635

76. Ramakrishnan VR, Holt J, Nelson LF, Ir D, Robertson CE, Frank DN. Determinants of the nasal microbiome: pilot study of effects of intranasal medication use. Allergy Rhinol. (2018) 9:2152656718789519. doi: 10.1177/2152656718789519

77. Ghogomu N, Kern R. Chronic rhinosinusitis: the rationale for current treatments. Exp Rev Clin Immunol. (2017) 13:25970. doi: 10.1080/1744666X.2016.1220833

78. Nayan S, Maby A, Endam LM, Desrosiers M. Dietary modifications for refractory chronic rhinosinusitis? Manipulating diet for the Modulation of Inflammation. Am J Rhinol Allergy. (2015) 29:e170-4. doi: 10.2500/ajra.2015.29.4220

79. Hutson K, Clark A, Hopkins C, Ahmed S, Kumar N, Carrie S, et al. Evaluation of smoking as a modifying factor in chronic rhinosinusitis. JAMA Otolaryngol Head Neck Surg. (2021) 147:159-65. doi: 10.1001/jamaoto.2020.4354

80. Ramakrishnan VR, Frank DN. Impact of cigarette smoking on the middle meatus microbiome in health and chronic rhinosinusitis. Int Forum Allergy Rhinol. (2015) 5:981-9. doi: 10.1002/alr.21626

81. Anderson M, Stokken J, Sanford T, Aurora R, Sindwani R. A systematic review of the sinonasal microbiome in chronic rhinosinusitis. 
Am J Rhinol Allergy. (2016) 30:161-6. doi: 10.2500/ajra.2016. 30.4320

82. Ba L, Zhang N, Meng J, Zhang J, Lin P, Zhou P, et al. The association between bacterial colonization and inflammatory pattern in Chinese chronic rhinosinusitis patients with nasal polyps. Allergy. (2011) 66:1296303. doi: 10.1111/j.1398-9995.2011.02637.x

83. Ahmed N, Mahmoud NF, Solyman S, Hanora A. Human nasal microbiome as characterized by metagenomics differs markedly between rural and industrial communities in egypt. Omics. (2019) 23:573-82. doi: 10.1089/omi.2019.0144

84. Stephenson MF, Mfuna L, Dowd SE, Wolcott RD, Barbeau J, Poisson $\mathrm{M}$, et al. Molecular characterization of the polymicrobial flora in chronic rhinosinusitis. J Otolaryngol Head Neck Surg. (2010) 39:182-7. doi: 10.2310/7070.2009.090060

85. Jiang RS, Su MC, Lin JF. Nasal mycology of chronic rhinosinusitis. Am J Rhinol. (2005) 19:131-3. doi: 10.1177/194589240501900204

86. Renteria AE, Mfuna Endam L, Desrosiers M. Do aging factors influence the clinical presentation and management of chronic rhinosinusitis? Otolaryngol Head Neck Surgery. (2017) 156:598-605. doi: 10.1177/01945998176 91258

87. Chong LY, Head K, Hopkins C, Philpott C, Glew S, Scadding G, et al. Saline irrigation for chronic rhinosinusitis. Cochrane Database Syst Rev. (2016) 4:Cd011995. doi: 10.1002/14651858.CD011995.pub2

88. Chong LY, Head K, Hopkins C, Philpott C, Schilder AG, Burton MJ. Intranasal steroids versus placebo or no intervention for chronic rhinosinusitis. Cochrane Database Syst Rev. (2016) 4:Cd011996. doi: 10.1002/14651858.CD011996.pub2

89. Khalil HS, Nunez DA. Functional endoscopic sinus surgery for chronic rhinosinusitis. Cochrane Database Syst Rev. (2006) 2006:Cd004458. doi: 10.1002/14651858.CD004458.pub2

90. Hoy SM. Dupilumab: a review in chronic rhinosinusitis with nasal polyps. Drugs. (2020) 80:711-7. doi: 10.1007/s40265-020-01298-9

91. Kartush AG, Schumacher JK, Shah R, Patadia MO. Biologic agents for the treatment of chronic rhinosinusitis with nasal polyps. Am J Rhinol Allergy. (2018) 33:203-11. doi: 10.1177/1945892418814768

92. Glück U, Gebbers J-O. Ingested probiotics reduce nasal colonization with pathogenic bacteria (Staphylococcus aureus, streptococcus pneumoniae, and $\beta$-hemolytic streptococci). Am J Clin Nutr. (2003) 77:517-20. doi: 10.1093/ajcn/77.2.517

93. Mukerji SS, Pynnonen MA, Kim HM, Singer A, Tabor M, Terrell JE. Probiotics as adjunctive treatment for chronic rhinosinusitis: a randomized controlled trial. Otolaryngol Head Neck Surgery. (2009) 140:2028. doi: 10.1016/j.otohns.2008.11.020

94. Cervin AU. The potential for topical probiotic treatment of chronic rhinosinusitis, a personal perspective. Front Cell Infect Microbiol. (2017) 7:530. doi: $10.3389 /$ fcimb. 2017.00530

95. Sur DK, Plesa ML. Treatment of allergic rhinitis. Am Family Phys. (2015) 92:985-92.

96. Shinee T, Sutikno B, Abdullah B. The use of biologics in children with allergic rhinitis and chronic rhinosinusitis: current updates. Pediatric Inv. (2019) 3:165-72. doi: 10.1002/ped4.12146

97. Lei DK, Saltoun C. Allergen immunotherapy: definition, indications, and reactions. Allergy Asthma Proc. (2019) 40:36971. doi: $10.2500 /$ aap. 2019.40 .4249

Conflict of Interest: The authors declare that the research was conducted in the absence of any commercial or financial relationships that could be construed as a potential conflict of interest.

Publisher's Note: All claims expressed in this article are solely those of the authors and do not necessarily represent those of their affiliated organizations, or those of the publisher, the editors and the reviewers. Any product that may be evaluated in this article, or claim that may be made by its manufacturer, is not guaranteed or endorsed by the publisher.

Copyright (๑) 2021 Huntley, Raber, Fine and Bernstein. This is an open-access article distributed under the terms of the Creative Commons Attribution License (CC BY). The use, distribution or reproduction in other forums is permitted, provided the original author(s) and the copyright owner(s) are credited and that the original publication in this journal is cited, in accordance with accepted academic practice. No use, distribution or reproduction is permitted which does not comply with these terms. 